pour ses membres de la souscription à la Bibliothèque d'Humanisme et Renaissance et à la Bibliographie Internationale d'Humanisme et de la Renaissance.

Des informations sur les activités locales et régionales de différents groupes sur la Renaissance sont présentées par le président, professeur Hoeniger (Edmonton et Toronto), par les professeurs Gary Waller (Dalhousie), Bergmann, Michael Best (U.B.C.) et De Bujanda (Sherbrooke). Le professeur Don Beecher présente un compte rendu de la réunion récente de la North Central Branch tenue à Cleveland et annonce que la prochaine rencontre aura lieu à l'Université Carleton en avril 1978. Il parle également du Centre de la Renaissance de Carleton. Le professeur De Bujanda prépare annuellement pour la Bibliographie Internationale d'Humanisme et de la Renaissance un résumé des publications sur la Renaissance parues au Canada.

Le professeur Bergmann propose une motion de reconnaissance au professeur Eva Kushner. La proposition est secondée par le professeur Mélançon et approuvée à l'unanimité.

Le professeur Lindeman propose une motion de reconnaissance au professeur Don Beecher pour le magnifique concert de musique de la Renaissance qu'il nous a offert. La motion a été secondée par tous ceux qui ont assité au concert.

En vue de la rencontre de l'année prochaine, le président suggère qu'il y ait une session consacrée à la Renaissance italienne et une autre à la Réforme. Il invite les membres à lui envoyer des suggestions concernant le programme et les autres activités de la Société. Il signale que la Société appuiera le projet-Gair de publication de la monographie s'il y a un nombre suffisant de collaborateurs enthousiastes. On devra contacter le professeur Gair personnellement.

L'assemblée exprime sa reconnaissance au professeur Gair pour le magnifique travail qu'il a fait pour la préparation et pendant les réunions de Frédericton.

La réunion est ajournée à 18 h.00

\title{
OLGA PUGLIESE
}

Rapports présentés à la réunion d'affaires du 28 mai 1977

(Annexe aux minutes de l'Assemblée générale tenue à l'Université du Nouveau-Brunswick, Frédericton)

\section{Rapport sur l'état des adhésions à la Société}

Au cours de sa première année d'existence, la Société a accueilli 135 
membres. Parmi ceux-ci, 13 sont des membres fondateurs. La répartition des membres par province est la suivante:

$\begin{array}{lr}\text { Colombie Britannique: } & 9 \\ \text { Alberta } & 12 \\ \text { Saskatchewan } & 3 \\ \text { Manitoba } & 2 \\ \text { Ontario } & 68 \\ \text { Québec } & 27 \\ \text { Nouveau-Brunswick } & 10 \\ \text { Nouvelle-Ecosse } & 2 \\ \text { Terre-Neuve } & 2 \\ \text { Ile-du-Prince-Edouard } & 0\end{array}$

En vue d'attirer de nouveaux membres, l'exécutif a fait publier des nouvelles sur la formation de la Société dans une douzaine de revues. Ces nouvelles sont déjà parues dans Humanities Research Council Bulletin, University of Toronto Staff Bulletin, Spenser Newsletter, Renaissance Quarterly et bien entendu dans Renaissance et Réforme.

\section{Rapport financier}

La cotisation annuelle pour l'année 1976-1977 était de \$10. pour les membres réguliers, $\$ 5$. pour les étudiants gradués et les professeurs à la retraite ainsi que pour les détenteurs d'un Ph.D. qui sont sans poste académique (ceux-ci forment une nouvelle catégorie dont le nombre s'accroît dans la situation actuelle) et entre $\$ 50$. et $\$ 100$. suggéré comme don pour les membres fondateurs. Il avait été décidé que les argents offerts par les membres fondateurs seraient gardés dans un fond de la Société pour des fins spécifiques (non pas pour payer les dépenses de ses membres par exemple). Il faut signaler que les membres fondateurs devront renouveler leur cotisation pour l'année 1977-78 et payer le montant régulier.

Sur cette base, les revenus provenant des cotisations des membres s'élèvent à $\$ 1,970.00$ (jusqu'à présent, on a reçu $\$ 2,137$. mais il faut déduire de ce montant $\$ 167$. qui correspondent à des montants payés en trop et principalement à des souscriptions à la revue).

De ces $\$ 1,970.00, \$ 700.00$ proviennent des contributions des membres fondateurs. Il nous reste donc une balance de $\$ 1,270.00$.

Liste des déboursés: $\$ 88.67$ fourniture et matériel (poste, xérox)

2.00 frais bancaires

300.00 contribution à la revue Ren. \& Ref.

116.00 dépenses pour la rencontre du sous-

$\$ 506.67$ Total 


\section{2 / Renaissance and Reformation}

La Société dispose maintenant d'un solde de $\$ 763$. (plus $\$ 700$. dans des fonds spéciaux).

N.B. Les dépenses pour la réunion du comité exécutif du mois d'octobre s'élevaient à $\$ 844.79$. et furent couvertes par une subvention du Conseil des Arts qui était de $\$ 1,896$. dont $\$ 1,051.21$ ont été retournés.

Rapport des décisions prises par l'exécutif pendant l'année 1976-1977

1. La Revue. En septembre 1976 s'est réuni un sous-comité pour discuter des problèmes concernant la revue Renaissance and Reformation dont les recommandations furent acceptées par l'exécutif en octobre. On a accepté que la Société devienne co-patron de la revue, avec North Central Conference of the Renaissance Society of America, et avec les premiers patrons, soient Toronto Renaissance and Reformation Colloquium et Victoria University Centre for Reformation and Renaissance Studies. A compter du premier tirage de 1977 (à paraître le 1er juillet), la revue aura une couverture et un titre bilingue (Renaissance and Reformation: Renaissance et Réforme) dans lequel paraîtront des articles en français et en anglais ainsi que de temps à autre des informations concernant la S.C.E.R./C.S.R.S.

Le nouvel éditeur de la revue, qui succède à Julius A. Molinaro, est Richard Van Fossen de l'Université de Toronto et l'éditeur-adjoint, André Berthiaume de l'Université Laval. La S.C.E.R. a deux représentants officiels au comité de rédaction, J.M. De Bujanda de l'Université de Sherbrooke et S.K. Heninger de U.B.C. Claude Sutto (Montréal) est associé à Ian Lancashire de Toronto pour les compte-rendu de volumes.

Le prochain numéro de la revue qui paraîtra vers le 1 er juillet contiendra le texte de la constitution de la Société dans les deux langues.

Le rapport financier signale que la S.C.E.R. a accordé une somme de $\$ 300$. en tant que co-patron pour l'année 1976-1977. Les sommes a être accordées à l'avenir seront déterminées en tenant compte de la situation financière de la revue.

Notre co-patronnage a eu aussi comme résultat d'augmenter le nombre des souscriptions à la revue puisque approximativement 40 de nos membres ont souscrit au journal par notre intermédiaire.

2. L'Affiliation à d'autres sociétés. Pour ce qui se rapporte au Conseil Canadien de Recherche sur les Humanités du Canada, nous avons été informés que nous pourrons déposer une demande d'application seulement après trois ans d'existence de notre Société, ce qui nous reporte à notre réunion annuelle de 1979.

Le comité exécutif a décidé de faire application pour l'affiliation de la Société à la Fédération Internationale des Sociétés et Instituts pour 
l'Etude de la Renaissance. On a déjà établi certains contacts avec le professeur Halkin, président de la Fédération (on lui a déjà envoyé les informations sur notre Société).

3. Comité de nomination. Pour préparer notre rencontre de l'année prochaine à l'Université de Western Ontario, le comité exécutif à mis sur pied un comité de nomination qui devra présenter une liste des personnes proposées pour former le comité exécutif et le conseil pour le terme 1978-1980. Les membres proposés pour faire partie du comité de nomination sont les suivants:

Harry Secor (Département de français, Un. de Toronto), président Claude Sutto (Histoire, Univ. de Montréal)

James Black (Département d'anglais, Un. de Calgary)

Mary Ella Milham (Département des études classiques, Un. du N.B.)

\section{North Central Conference of the Renaissance Society}

The Coordinating Secretary reports that the complexities of crosschecking have delayed the report on teaching activities; a full report will appear in Volume XIV, Number 1. 